\title{
Effect of oral high frequency ventilation by jet or oscillator on minute ventilation in normal subjects
}

\author{
RJD GEORGE, RJD WINTER, MA JOHNSON, IP SLEE, DM GEDDES
}

From the London Chest Hospital and St Stephen's Hospital, London

\begin{abstract}
Normal subjects were asked to breathe through an open ended tube while high frequency oscillations were superimposed on tidal breathing via a side arm, either an eight inch $(20 \mathrm{~cm})$ loudspeaker or a jet ventilator being used. Both systems were comfortable and well tolerated. Spontaneous minute ventilation fell by $19-46 \%$ at frequencies up to $33 \mathrm{~Hz}$ without a rise in transcutaneous $\mathrm{PCO}_{2}$. Maximum ventilatory savings occurred at $1.6 \mathrm{~Hz}$ with the jet ventilator $(\mathrm{p}<0.01)$ and at a frequency corresponding to respiratory system resonance with the loudspeaker. This suggests that during oral high frequency ventilation pulmonary gas exchange is improved and leads to more efficient carbon dioxide excretion for a given minute ventilation. This technique provides a practical and simple method of supplementing breathing in conscious subjects, and it may also have application in the management of patients with acute or chronic respiratory failure, where intubation and conventional ventilation might be avoided.
\end{abstract}

Normal breathing requires the movement of a tidal volume at least twice that of the anatomical dead space. In the early 1970 s, however, two groups showed that it was possible to achieve successful ventilation in experimental animals and subsequently in man with volumes less than the conventionally measured anatomical dead space, provided that these volumes were delivered at frequencies exceeding about $1 \mathrm{~Hz} .^{12}$ This mode of ventilation has become known as high frequency ventilation (HFV) and in the past 10 years two principal techniques of delivery have evolved:

1 High frequency oscillation (HFO), in which sine wave oscillations of flow are produced in the airways with an eccentric cam piston or the diaphragm of a loudspeaker. ${ }^{34}$ Fresh gas passed across the endotracheal tube (a biased flow) serves to remove carbon dioxide and to provide fresh, oxygenated gas. There are no limitations to frequency with this system, although the quality of the waveform when a loudspeaker is used may be diminished below about $5 \mathrm{~Hz}$. Frequencies of up to $30 \mathrm{~Hz}$ have been used with success both in animal ${ }^{15}$ and in neonatal human studies. ${ }^{6}$

2 High frequency jet ventilation (HFJV) and high Address for correspondence: Dr RJD George, London Chest Hospital, London E2 9JX.

Accepted 15 April 1985 frequency positive pressure ventilation, in which the air is oscillated within the lungs by insufflating pulses of gas into the airway. Elastic recoil of the lung and chest wall provides the expiratory limb for each pulse. Use of these systems has been restricted to frequencies of $10 \mathrm{~Hz}$ and below and several ventilators using this principle have been designed. ${ }^{7-9}$

HFV is the subject of much research and a substantial body of publications has accumulated. Although the mode of action remains obscure, there are unlikely to be major differences between the two systems. ${ }^{10}$ It has, however, been established that HFV is an effective and safe mode of ventilatory support with various delivery systems. ${ }^{2} 1112$

Work so far has been confined largely to the use of the technique as an alternative to low frequency, high volume positive pressure ventilation, yet a prominent and well described feature of HFV is that spontaneous breathing is unhindered. ${ }^{11-13}$ If HFV could be delivered without intubation, the technique might have considerable application in the management of patients with varying degrees of respiratory failure. There are very few published data on the application of HFV in this area, although recently high frequency oscillation using external chest wall compression has been shown to reduce minute ventilation in animals and man. ${ }^{14}$ is

In this study we report the effects of oral HFV on breathing in normal subjects at rest using oral high 
frequency oscillations (OHFO) and oral high frequency jet ventilation (OHFJV).

\section{Methods}

We studied two groups of seven healthy men (mean age 33 years). None smoked and all had normal lung function as assessed by spirometry and carbon monoxide transfer.

Two studies were performed to observe the effect on breathing of HFV delivered through a conventional mouthpiece-one using a high frequency jet ventilator and the other using an oscillator. The physiological monitoring methods were common to both studies. Ventilation (VE) was measured with an inductance plethysmograph (Respitrace). This had been validated against a pneumotachograph in a preliminary study. The mean tidal volume (VT) (668 ml) measured by the two techniques showed no significant difference (mean difference $23 \mathrm{ml}$, two tailed $t$ test). Measurements in DC mode allowed estimation of any change in functional residual capacity (FRC). The Respitrace chest and abdominal bands were attached firmly to the skin with adhesive tape to prevent slipping. Calibration was performed at the beginning and end of each study with the isovolume manoeuvre, ${ }^{16}$ the subject being seated as for the experiment. Transcutaneous carbon dioide partial pressure $\left(\mathrm{TcPCO}_{2}\right)$ was monitored continuously with a miniaturised Stow-Severinghaus electrode (Radiometer TCM20); previous studies had shown this to be a reliable index of changes in arterialised capillary blood $\left(\mathrm{tcPCO}=0.93 \mathrm{PaCO}_{2}+\right.$ $10.8 ; \mathrm{r}=0.84, \mathrm{p}<0.001)$. The electrode was calibrated at the beginning and end of each study according to the manufacturer's instructions with standard $5 \%$ and $10 \%$ carbon dioxide reference gas.

Figure 1 shows the experimental arrangement in both the OHFO and the OHFJV studies and indicates the measurements made.

\section{EQUIPMENT}

Mean tracheal pressures around $500 \mathrm{~Pa}\left(5 \mathrm{~cm} \mathrm{H}_{2} \mathrm{O}\right)$ are usually quoted in publications on anaesthesia when HFV is used as an alternative to conventional ventilation. ${ }^{71112}$ With such pressures, delivery of cold, dry gas directly into the mouth leads to local irritation and oscillation of the mobile structures-a problem aptly termed disco uvula. ${ }^{17}$ As the intention of the present study was to supplement rather than abolish spontaneous breathing, mouth pressures were reduced and pressures of 126-146 $\mathrm{Pa}$ (1.3-1.5 $\mathrm{cm} \mathrm{H}_{2} \mathrm{O}$ ) were found to be acceptable.

\section{High frequency jet ventilators}

Each subject breathed through an open ended tube

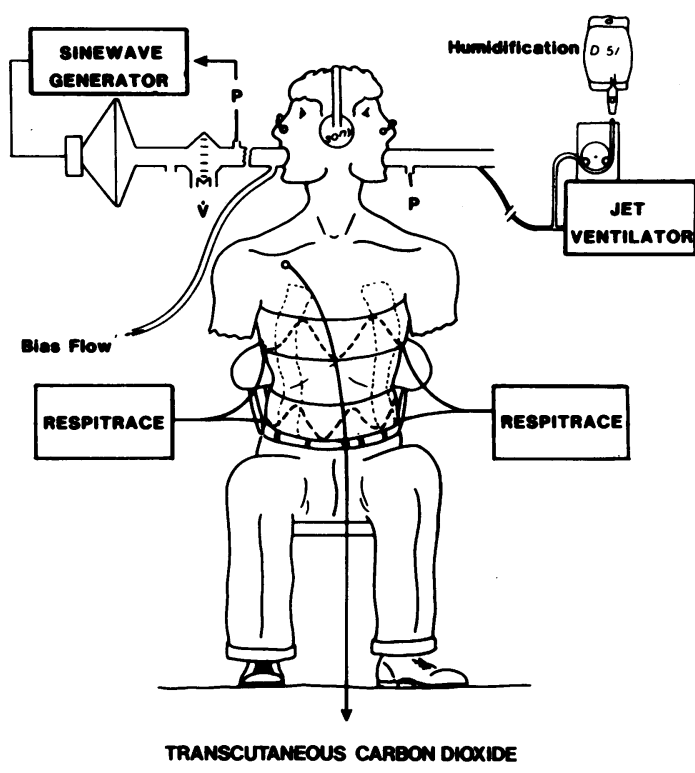

Fig 1 The experimental setup for oral high frequency oscillations and oral high frequency jet ventilation (see text).

(internal diameter $2.5 \mathrm{~cm}$ ). To overcome the local trauma from a jet delivered directly into the mouth, the high pressure jet entered the side wall of the breathing tube (volume $170 \mathrm{ml}$ ) at an angle of $45^{\circ}$ through a $2 \mathrm{~mm}$ orifice. Thus the narrow stream struck the opposite wall to develop a pulsetrain atraumatically. The additional dead space also accommodated initial cooling and drying of the jet air as it emerged from the small orifice. Two ventilators were used in the study: a commercial model (Acutronic 800), which delivered frequencies up to $10 \mathrm{~Hz}$; and a homemade ventilator used to deliver additional frequencies of $16.5 \mathrm{~Hz}$ and $33 \mathrm{~Hz}$, at the same pressure through the same tubing. Both systems were based on a solenoid valve. The mean input pressure, measured at the mouth by a Gaeltec D360 transducer, was maintained at $146 \mathrm{~Pa}(1.5 \mathrm{~cm}$ $\mathrm{H}_{2} \mathrm{O}$ ). Each jet pulse occupied half of the overall cycle time and the subject continued to breathe through the open ended mouthpiece (fig 1).

\section{High frequency oscillator}

Sinewave oscillations were generated by a standard 8 inch $(20 \mathrm{~cm})$ bass loudspeaker connected to a mouthpiece by $50 \mathrm{~cm}$ length of semirigid tube (internal diameter $2.5 \mathrm{~cm}$ ). A small side arm near the loudspeaker allowed normal breathing of fresh air. Positioned between this side arm and the subject was a Fleisch pneumotachograph and differential pressure transducer (Gaeltec B681), from which estimates of instantaneous oscillatory flow rates 
( $\dot{V}_{\text {osc }}$ were made. The volume delivered by the oscillator (Vosc) was calculated by integration of the instantaneous flow signal tracings. A $1 \mathrm{~Hz}$ high pass filter was used to minimise the flow signal from tidal breathing. Oscillatory input pressure was maintained constant (mean pressure zero, root mean square input pressure $\left.126 \mathrm{~Pa}\left(1.3 \mathrm{~cm} \mathrm{H} \mathrm{H}_{2} \mathrm{O}\right)\right)$. This was monitored by an additional Gaeltec transducer between the pneumotachograph and the subject and fed back via a compressing amplifier ( $B$ and $K$ 2603) to maintain the oscillatory pressure constant. Flow impedance (peak input pressure/peak oscillatory flow) could therefore be expressed as a function of frequency. The dead space of the apparatus was reduced by a $41 / \mathrm{min}$ suction bias flow at the mouth. This did not influence measurements of flow or pressure.

\section{Calibrations}

The frequency responses of both pressure and flow transducers were measured by a technique similar to that used by Jackson and Vinegar. ${ }^{18}$ Pressure oscillations were produced in a closed tube (length $20 \mathrm{~cm}$, diameter $2.5 \mathrm{~cm}$ ) by the 8 inch speaker. The root mean square sound pressure was monitored by a standard, independently calibrated transducer (B and $K$ 4045) and compressing amplifier (B and $K$ 2603). By connecting this to the speaker amplifier we controlled its output to maintain the pressure in the tube constant over the frequency range 5-120 $\mathrm{Hz}$. Both transducers had flat frequency responses (coefficient of variation $<5 \%$ ) within this range compared with the standard at $2.0,80$, and $126 \mathrm{~Pa}$. Both transducers were subsequently calibrated against an inclined water manometer at the beginning and end of each study. To maximise the frequency response of the system, the connecting tubing on either side of the pneumotachograph was symmetrical and the tubing between the transducer and pneumotachograph was restricted to $1 \mathrm{~cm}$. The frequency response of the pneumotachograph and equipment as a whole was tested by attaching an Helmholz resonator (vol 5.61 l) to the mouthpiece. Measured flow impedance (peak input pressure/ peak input Vosc) was then compared with calculated impedance. The system was found to be linear to 30 $\mathrm{Hz}$ (mean measured impedance $101 \%$ of calculated impedance, coefficient of variation $7.7 \%$ ).

\section{EXPERIMENTAL PROCEDURE}

Animal work has suggested a complex relationship between carbon dioxide clearance, oscillatory flow, and volume, ${ }^{4} 1019$ and for reasons of comfort input pressures at the mouth in these studies were restricted. It therefore seemed important as part of the oscillator studies to investigate frequencies where flow rate would be maximum for a given input pressure (that is, at the resonant frequency, where changes in flow in response to pressure oscillations occur simultaneously and are said to be in phase). Resonant frequency was therefore found by displaying the input pressure and flow signals on an $X-Y$ oscilloscope to find the frequency at which flow and pressure were in phase. This was accompanied by a sensation of maximum shaking within the chest, which confirmed subjectively that flow was greatest at that frequency. The following frequencies were studied: OHFJV-Acutronic 800: 1.6, 3.3, 5, 8.3, and $10 \mathrm{~Hz}$; custom built solenoid ventilator 16.5 and $33 \mathrm{~Hz}$. OHFO-5, 10,15,20, and $33 \mathrm{~Hz}$, and respiratory system resonance (mean 7.5 , range $6.9-8 \mathrm{~Hz}$ ).

The experimental protocol was identical for the two studies. The subject was seated comfortably and listened to music by headphones. After a 10 minute acclimatisation period 10 minutes of HFV alternated with a five minute control period. Frequencies were applied in random order. Five minutes' control was found to be sufficient to allow tcPCO $\mathrm{CO}_{2}$ and $\mathrm{VE}$ to return to stable levels. Control periods were therefore restricted to five minutes to prevent the experiment from being unacceptably long.

DATA ANALYSIS

Minute ventilation; mean tidal volume; and inspiratory, expiratory, and total breath time ( $T$, TE, TTOT respectively) were measured during the last four minutes of each period of HPV. The control was taken as the mean of the last minute of every control period. Data on the acclimatisation period were not analysed.

Statistical analysis was by Wilcoxon's rank sum tests. Other methods are mentioned where appropriate in the text.

\section{Results}

Figure 2 shows a Respitrace recording at the end of OHFJV at $5 \mathrm{~Hz}$ and the beginning of a control period. This shows visually the oscillations produced by the jet superimposed on tidal breaths - that is, the subject's spontaneous $\dot{V} E$ and the additional ventilation available from the OHFJV.

Figure 3 is the $\mathrm{tcPCO}_{2}$ record of a complete study on a naive subject. The actual $\dot{V}_{E}$ for the last four minutes of each period is also shown. This demonstrates two features: an initial fall in $\mathrm{tcPCO}_{2}$ with a return towards normal as $\dot{\mathrm{V}}_{\mathrm{E}}$ falls and the reduction in these fluctuations as the subject responds more briskly to the OHFV. This is highlighted by the repeat of the $3.3 \mathrm{~Hz}$ frequency at the end of the study, where VE fell by a similar amount but $\mathrm{tcPCO}_{2}$ 


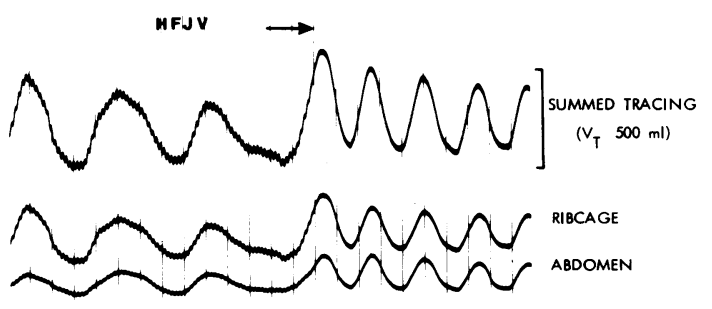

Fig 2 Record of tidal breathing monitored by Respitrace at the end of a period of oral high frequency jet ventilation. It shows the oscillations delivered by the ventilator superimposed on spontaneous tidal breathing. When the device is switched off there is a rapid change in breathing pattern, although the relative contributions of ribcage and abdomen are unchanged. Each vertical line represents one second.

fell only $3 \mathrm{~mm} \mathrm{Hg}$ compared with $10 \mathrm{~mm} \mathrm{Hg}$ on the first occasion. Despite the transient changes seen in $\mathrm{tcPCO}_{2}$ there was no overall change with either form of oscillation. Data on $\mathrm{tcPCO}_{2}$ were available in only four subjects using OHFJV-see figure 4, which summarises the results for both OHFO and OHFJV. There was a reduction in mean VE expressed as a percentage of control values at all frequencies studied with both techniques $(\mathrm{p}<0.05)$. Reductions with OHFJV exceeded those of OHFO at the higher frequencies $(p<0.05)$. The maximum fall in $\dot{V}_{E}$ occurred at $1.6 \mathrm{~Hz}$ with the jet ventilator, when VE fell to $54 \%$ (range $38-60 \%$ ) of the control value. It was not possible to obtain comparable data at this frequency for OHFO. Although the reduction in $\dot{V} E$ was maximum at the respiratory flow resonant frequency, it was not strikingly more effective than 15 $\mathrm{Hz}$ or above $(\mathrm{p}<0.05$, fig 4$)$.
Absolute changes in oscillatory flow and volume during OHFO are shown in figure 5 . Vosc by definition was maximum at the flow resonant frequency (mean $7.5 \mathrm{~Hz}$ ), where the mean value of impendance was $0.32 \mathrm{kPa} . \mathrm{l}^{-1} \cdot \mathrm{s}^{-1}$ This agrees with the results established from earlier studies. ${ }^{20}$ In five of the subjects the fall in $\dot{V} E$ was related to $\dot{V}$ osc and log Vosc. The rank correlations (Spearman) for these varied between individuals from $0.73(\mathrm{p}<$ $0.01)$ to $0.98(\mathrm{p}<0.001)$.

\section{CHANGES IN BREATHING PATTERN}

The rapid change in breathing pattern when OHFJV was stopped is seen in figure 2 . The mean changes in six subjects are shown as a spirogram in figure 6 . The mean respiratory cycle time is plotted against tidal volume for control and for OHFO $(7.5 \mathrm{~Hz})$ and OHFJV $(8.3 \mathrm{~Hz})$; TE was taken as the time for expiration to reach FRC. The pause at FRC has been plotted as a straight line at zero VT. With both modes of OHFV there was a significant reduction in inspiratory flow rate as shown by a fall in VT/TI. This was due to an increase in TI rather than a fall in VT. In both groups during OHFV there was also substantial lengthening of TTOT due both to a prolonged TE $(p<0.05)$ and to an increase in the end expiratory pause $(\mathrm{p}<0.05)$.

The Respitrace in DC mode showed no sustained or consistent change in FRC and the relative contributions of chest and abdominal components stayed the same (fig 2).

\section{Discussion}

\section{CHANGES IN VENTILATION}

There were significant reductions in resting $\dot{V} E$ at all frequencies with both modes of OHFV. The greater

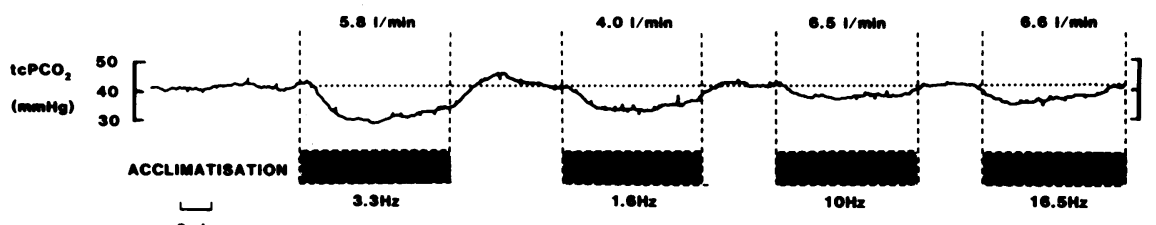

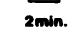

TIME

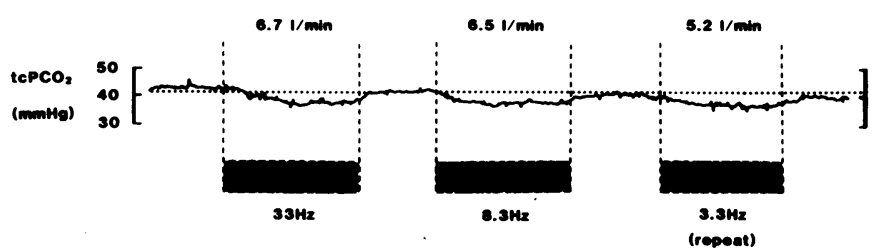

Fig 3 A continuous tracing from one study using oral high frequency ventilation in a naive subject for the first time. The mean minute ventilation for the last four minutes of each period is shown above the transcutaneous carbon dioxide pressure ( $\mathrm{tcP} \mathrm{CO}_{2}$ ) trace. The mean control minute ventilation in this study was $8.31 . \mathrm{min}^{-1}$ (see text). 

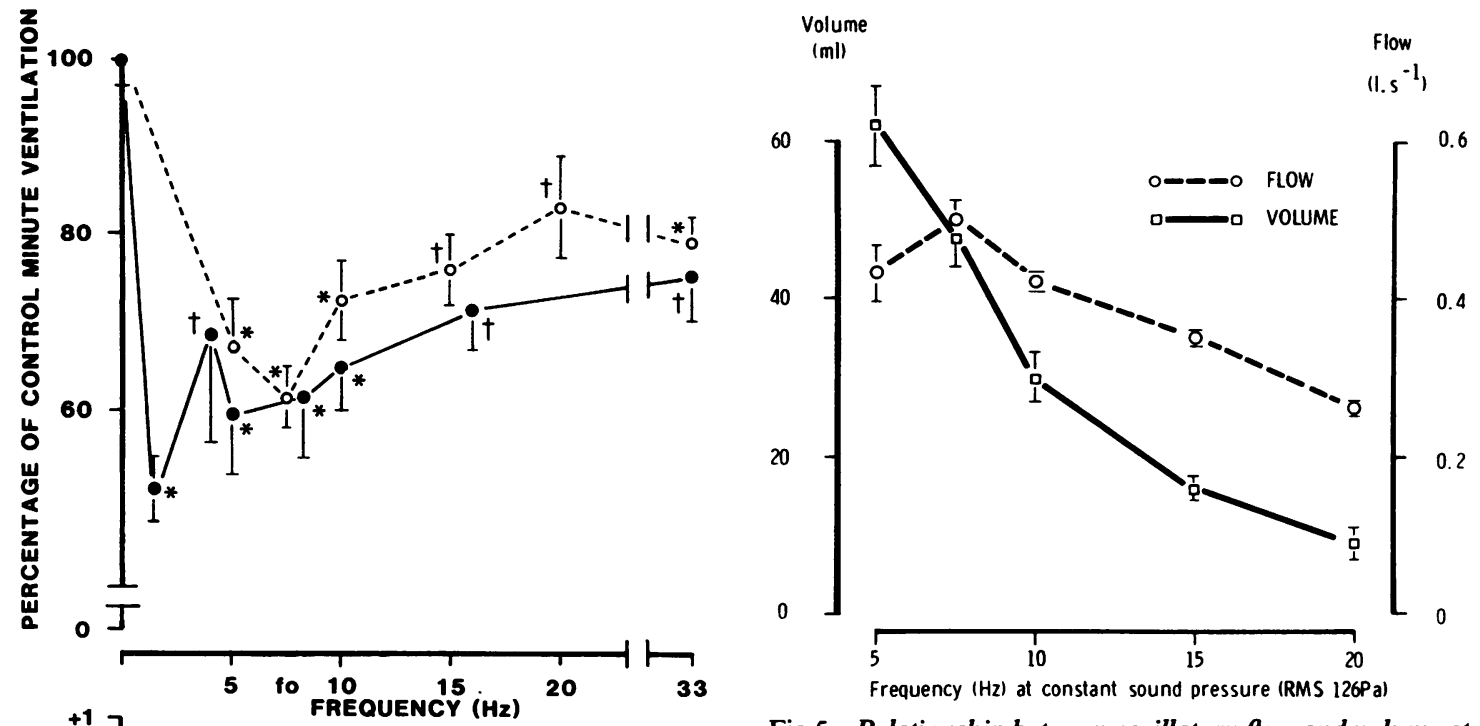

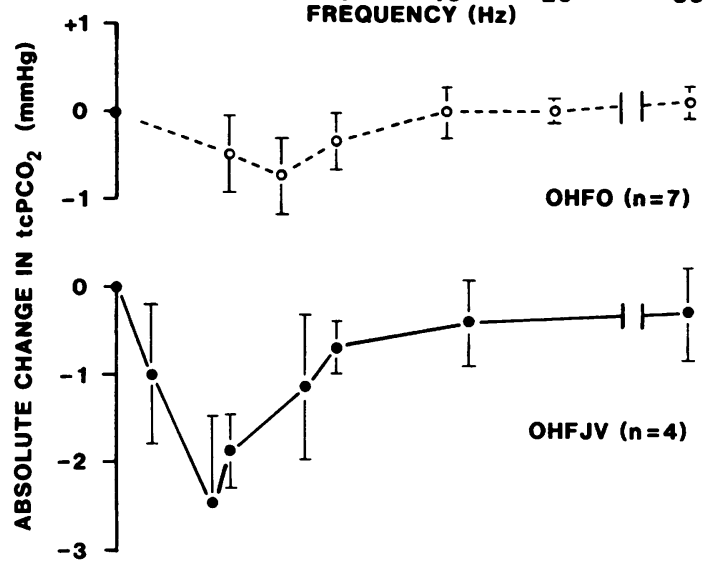

Fig 4 Upper panel: Reduction in minute ventilation during both oral high frequency oscillations $(O H F O)\left(O-O^{-}\right)$and oral high frequency jet ventilation $(O H F J V)(-0)$ plotted against frequency. The changes in minute ventilation have been expressed as a mean percentage of control values to allow for the variation in baseline levels between subjects. ${ }^{*}$ denotes significant differences at $p<0.01$ and $\dagger$ denotes significant differences at $p<0.05$ by comparison with control values. The bars show the standard errors. " $f o$ " represents the resonant frequency. The lower two panels show the changes in transcutaneous carbon dioxide pressure $\left(t c P\left(\mathrm{O}_{2}\right)\right.$ expressed as absolute changes from the baseline.

savings obtained with OHFJV at 16.5 and $33 \mathrm{~Hz}$ may have been a function of the higher input pressures (OHFJV mean input pressure $146 \mathrm{~Pa}$, OHFO mean input pressure $126 \mathrm{~Pa}$.), when the delivered ventilator flow and volumes are somewhat larger, or may have been due to other differences in the two techniques, such as the waveform or pressure.
Fig 5 Relationship between oscillatory flow and volume at different frequencies during oral high frequency oscillation. The points are mean values with $1 S D$.
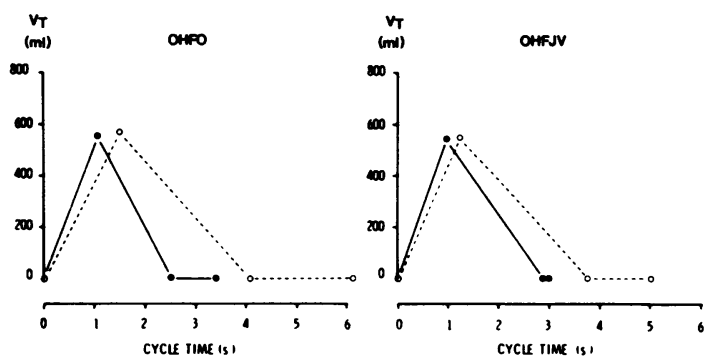

Fig 6 Mean changes in breathing pattern in six subjects from each group. The panels show a schematic spirogram comparing mean values for tidal volume (VT) and the respiratory cycle time during control periods (-) and oral high frequency oscillations $(O H F O)$ at the resonant frequency (mean $7.5 \mathrm{~Hz}$ ) and oral high frequency jet ventilation $(\mathrm{OHFJV})$ at $8.3 \mathrm{~Hz}(\mathrm{O}-\mathrm{O})$.

Although the delivery systems we used have tended to emphasise the similarities between the delivery of a pulsed and oscillating volume of gas, the inspiratory phase of the jet, particularly below $10 \mathrm{~Hz}$ when measured at the mouth, corresponds more to a square wave than to a sine wave. In addition, in contrast to OHFO, there is no negative pressure phase during OHFJV and the expiratory part of the oscillation is provided by elastic recoil of the respiratory system. The effect of different pressure waveforms during HFV is not established, but the maintenance of a positive end expiratory pressure may be important. ${ }^{11} 21$ There were, however, no sustained changes in FRC during any of the studies. 
POSSIBLE MECHANISMS OF REDUCTION OF VENTILATION

There were no sustained changes in $\mathrm{tcPCO}_{3}$ during either study despite a substantial fall in VE. This suggests that OHFV was supplementing alveolar ventilation and that these normal subjects responded to the tendency for carbon dioxide tension to fall by suppressing spontaneous ventilation to maintain normal levels within close limits. The nature of this response is seen in figure 3 , which documents a whole study of one subject during OHFJV. It serves to illustrate two points. Firstly, in all subjects $\mathrm{tcPCO}_{2}$ fell at the onset of oral HFV and subsequently, as VE was reduced, the $\mathrm{tcPCO}_{2}$ returned towards baseline levels. Secondly, as the subject "acclimatised" to the effect of OHFJV reduction in $\dot{V}_{E}$ was more rapid, so that the fluctuations in $\mathrm{tcPCO}_{2}$ were less evident. This is shown in figure 3 , where the fall in $\mathrm{tcPCO}_{2}$ in response to OHFJV at $3.3 \mathrm{~Hz}$ was substantially less by the end of the study.

Goldstein et $a^{22}$ measured carbon dioxide clearance by OHFO during voluntary breath holding with an open glottis in normal subjects. They found that it increased with Vosc, Vosc and lung volume, which is consistent with our findings. Since any reduction in spontaneous $\dot{V}_{E}$ during the studies reported here would almost certainly have been at the expense of alveolar ventilation, one may estimate the additional carbon dioxide clearance provided by OHFO. From our raw data the mean calculated alveolar ventilation was $5.21 . \mathrm{min}^{-1}$ (estimated on the basis of a $\mathrm{PaCO}_{2}$ of about $33 \mathrm{~mm} \mathrm{Hg}$ and carbon dioxide production of $200 \mathrm{ml} / \mathrm{min}) .^{23}$ To maintain isocapnia, the reductions in $\dot{V} E$ seen at 10 $\mathrm{Hz}$ in our subjects (range 0.3-4.3 $1 . \mathrm{min}^{-1}$ ) would have required an additional mean carbon dioxide clearance of 100 (range 10-163) ml. $\mathrm{min}^{-1}$. Vosc was $30 \mathrm{ml}$ and measurements made by Goldstein et $a l^{22}$ at $10 \mathrm{~Hz}$, using this oscillatory volume, showed values of carbon dioxide elimination ranging from 10 to 160 (mean 78 ) ml.min ${ }^{-1}$. Given changes in lung volume with spontaneous breathing, differences in equipment, and the assumptions made, these numbers are in reasonable agreement.

Although a small reduction in carbon dioxide production must accompany the fall in $\dot{V} E$, it is almost certainly insignificant, since under resting conditions the work of breathing normally takes no more than $2 \%$ of oxygen consumption. ${ }^{23}$

With both systems the reduction in $\dot{V}_{E}$ was associated with both a fall in VT/TI and prolongation of TE (fig 6). If these changes reflect a reduction in output of the respiratory centre,$^{24}$ then this may be attributable either to the transient changes in $\mathrm{PaCO}_{2}{ }^{25}$ or to an additional increase in afferent nervous discharge. There is good evidence from animal work that alterations in breathing pattern during HFV are influenced by stimulation of the pulmonary stretch receptors. A recent study in anaesthetised dogs, where lung volumes and blood gas tension were kept constant, showed a prolongation of TE during HFV similar to the values in our study, although the airway pressures were much greater. ${ }^{26}$ The prolongation was abolished by vagotomy. Other work showed a reduction in phrenic nerve discharge during HFV, which was also abolished by vagotomy. ${ }^{27}$ Figure 3 shows a typical tracing at the end of a run of OHFJV, where there is an immediate change in the breathing pattern towards normal. This rapid change is consistent either with sensing of change in $\mathrm{PaCO}_{2}$ oscillations or with a change in afferent discharge.

\section{PRACTICAL IMPLICATIONS}

HFV by jet or oscillator adapted for oral delivery provides a comfortable and simple method of supplementing voluntary breathing in normal subjects without the need for intubation. The improved carbon dioxide clearance leads to a reduction in minute ventilation rather than change in blood gas tensions. There may therefore be a role for this technique as support for patients who are unable to maintain adequate carbon dioxide clearance owing to exhaustion or weakness and where intermittent positive pressure ventilation would be undesirable. This technique might also have an application in the relief of breathlessness or the improvement of exercise tolerance. The encouraging results in normal subjects merit extension of this work to patients with breathing difficulties.

We wish to thank Dr RC Schroter for his help during the preparation of this manuscript. This study was funded by the Medical Research Council.

\section{References}

1 Lunkenheimer PP, Rafflenbeul W, Keller H, Frank H, Dickhut HH, Fuhrman C. Application of transtracheal pressure oscillations as a modification of diffusion respiration. $\mathrm{Br} J$ Anaes 1972;44:627.

2 Sjostrand UH, Eriksson W. High rates and low volumes in mechanical ventilation-not just a matter of ventilatory frequency. Anesthes Analges 1980;59: 567-76.

3 Bohn DJ, Mijasaka K, Marchac BE, Thompson WK, Froese AB, Bryan AC. Ventilation by high frequency oscillation. J Appl Physiol 1980;48: 710-6. 
4 Slutsky AS, Kamm RD, Rossing TH, Loring SA, Lehr J, Shapiro AH, Drazen JM. Effect of frequency, tidal volume and lung volume of $\mathrm{CO}_{2}$ clearance in dogs by high frequency oscillation $(2-30 \mathrm{~Hz}$ ) low tidal volume ventilation. J Clin Invest 1981;68:1475-84.

5 McEvoy RD, Davies NJH, Mannio FL, et al. Pulmonary gas exchange during high frequency ventilation. $J$ Appl Physiol 1982;52:1278-87.

6 Marchak BE, Thompson WK, Duffty P, et al. Treatment of RDS by high-frequency oscillatory ventilation a preliminary report. J Pediatr 1981;99:287-92.

7 Carlon GC, Miodownik S, Ray C, Kahn R. Technical aspects and clinical implications of high frequency ventilation with a solenoid valve. Crit Care Med 1981;9:47-50.

8 Borg U, Eriksson I, Sjostrand U. High frequency positive pressure ventilation (HFPPV): a view based upon its use during bronchoscopy and for laryngoscopy and microlaryngeal surgery under general anaesthesia. Anesthesanalgesia 1980;59:594-605.

9 Whitwam JG, Chakrabarti MK, Konarzewski WH, Askitopoulou H. A new valveless all-purpose ventilator. Br J Anaesth 1983;55:1017-23.

10 George RJD, Geddes DM. High Frequency Ventilation. Br J Hosp Med 1985;33:344-9.

11 Carlon GC, Kahn RC, Howland WS, Ray C, Turnbull AD. Clinical experience with high frequency jet ventilation. Crit Care Med 1981;9:1-6.

12 Klain M, Smith RB. High frequency percutaneous transtracheal jet ventilation. Crit Care Med 1977; 6:280-7.

13 Meeuwis H, Vaes L, Klain M. Long term high frequency ventilation in a 3 yr old child. Crit Care Med 1983;11:309-10.

14 Zidulka A, Gross D, Minami H, Vartian V, Chang HK. Ventilation by high frequency chest wall compression in dogs with normal lungs. Am Rev Respir Dis 1983;127:709-13.

15 Calverley PMA, Chang HK, White D, Zidulka A.
Effects of high frequency chest wall compression in normal subjects and COPD patients. Thorax 1984;39:235 (abstract).

16 Konno K, Mead J. Measurement of the separate volume changes of the ribcage and abdomen during breathing. J Appl Physiol 1967;22:487-522.

17 Froese AB, Bryan AC. High frequency ventilation. Am Rev Respir Dis 1981;123:249-50.

18 Jackson AC, Vinegar A. A technique for measuring the frequency response of pressure, volume and flow transducers. J Appl Physiol 1979;47:462-7.

19 Solway J, Gavriely N, Kamm RD, et al. Intra-airway gas mixing during high frequency ventilation. J Appl Physiol 1984;56:343-54.

20 Clement J, Landser FJ, Van de Woestijne KP. Total resistance and reactance in patients with respiratory complaints with and without airway obstruction. Chest 1983;83:215-20.

21 Gallagher TJ, Klain MM, Carlon GC. Present status of high frequency ventilation. Crit Care Med 1982; 10:613-7.

22 Goldstein D, Slutsky AS, Ingram RH, Westerman P, Venegas J, Drazen J. $\mathrm{Co}_{2}$ elimination by high frequency ventilation $(4-10 \mathrm{~Hz})$ in normal subjects. $A m$ Rev Respir Dis 1981;123:251-5.

23 Cotes JE. Lung function: principles and application in medicine. 4th ed. Oxford: Blackwell Scientific Publications, 1979:387.

24 Clark FJ, von Euler C. On the regulation of the depth and rate of breathing. J Physiol 1972;222:267-95.

25 Semple SJG. The role of oscillations in arterial $\mathrm{Co}_{2}$ tension in the chemical control of breathing at rest and on exercise. Clin Sci 1984;66:639-42.

26 Banzett R, Lehr J, Geffroy B. High frequency ventilation lengthens expiration in the anaesthetised dog. $J$ Appl Physiol 1983;55:329-34.

27 Man GCW, Man FP, Kappagoda CT. Effect of high frequency oscillating ventilation on vagal and phrenic nerve activities. J Appl Physiol 1983;54:502-7. 